\title{
Spasticity Health Literacy Among Canadian Family Physicians
}

\author{
Joshua Goldstein, Chetan P. Phadke, Farooq Ismail, Chris Boulias
}

Keywords: botulinum toxin, muscle spasticity, family physicians, baclofen, health literacy

doi:10.1017/cjn.2015.271

Can J Neurol Sci. 2015; 42: 450-453

One of the consequences of upper motor neuron disease is the development of spasticity, classically defined as a "velocitydependent increase in muscle tone and hyper-excitability of the stretch reflex." Although alternative definitions have been recently proposed that define spasticity as a "disordered sensorimotor control," in this study, we focused on the classic definition underlying velocity dependence and hyperexcitability of stretch reflexes that is accepted and included in standard medical textbooks. Patients presenting with spasticity must first be assessed in a comprehensive manner to determine whether treatment is required or appropriate. The presence of spasticity may improve functionality by enabling standing, walking, and transferring. When spasticity is considered disabling and involves severe functional problems, management is essential to prevent further deterioration in function. If not managed in a timely manner, spasticity can lead to diminished activity and eventually can result in contractures.

There are several pharmacological approaches based on the presentation of spasticity. Oral medications such as baclofen and tizanidine have been proven to be effective for spasticity that is seen in multiple muscles and limbs (generalized spasticity). Although effective, oral medications have known dose-related side effects such as drowsiness, headache, nausea, and constipation. As a result, patients are often taken off these medications before a clinical improvement in their spasticity can be observed. ${ }^{2}$ Generalized spasticity that has proven unresponsive to oral baclofen or tizanidine is best treated with an intrathecal baclofen pump. ${ }^{2}$ Intramuscular injections of botulinum toxin have been repeatedly demonstrated in the literature to be effective for treating focal spasticity such as seen in the wrist and finger flexor muscle groups. ${ }^{2}$ This medication also bypasses the side effects described previously that are associated with systemic therapies. The decrease in muscle tone achieved with botulinum toxin can last for three to four months, and may be prolonged for even longer with the adjunctive use of physical therapy programs. ${ }^{2}$

The outcome of disabling spasticity is often a severe limitation of patients' activities of daily living, which can increase the burden on caregivers. This results in significant financial and social burdens for both the patient and the province in which the patient resides. Chronic spasticity can lead to a state of prolonged immobility, resulting in contractures, which further limit patient independence and add to caregiver requirements. ${ }^{2}$ It is for these reasons that appropriate medical care is paramount.

As is the case with most other chronic conditions, spasticity patients usually present to their family physicians in the initial stages of upper motor neuron syndrome. Because spasticity is the result of a variety of neurological and traumatic conditions, it is concerning that a previous study demonstrated limited knowledge in the recognition and treatment of spasticity among Ontario family physicians. ${ }^{3}$ Without this understanding, family physicians are not likely to appropriately refer and, therefore, without intention, deny the benefit of effective therapeutic intervention to the impaired patient. Given the importance and central role of the family physician in patient care, it is necessary to consider what factors may influence the physician's level of knowledge.

The purpose of this study was to assess spasticity-related literacy among family physicians across Canada and whether the literacy was related to gender, Canadian versus international medical training, and years of experience among family physicians.

\section{Methods}

This cross-sectional study was approved by the local hospital ethics board. An online Canadian medical directory, Scott's Medical Directory, was used to gather the email addresses of 2795 family physicians currently practicing in Canada. Physicians were categorized according to gender, years of experience, and location of training. Potential participants were emailed a questionnaire that was created by our group. The web portal Fluidsurvey.com was used to generate the online survey and send it out to the family physicians across Canada. A French translation of the survey was sent to those physicians located in Quebec. Physicians were informed that their confidentiality would be maintained and that completion of the questionnaire implied consent to participate in this study. Each question had room for additional written answers to reduce potential response bias created by using a multiple choice format. A follow-up email was sent to physicians who had not completed the survey after ten days. A second follow-up email was sent 20 days later as a final reminder to

From the Graduate Entry Medical School, University of Limerick, Limerick, Ireland (JG); Spasticity Research Program, West Park Healthcare Centre, and Department of Physical Therapy, Faculty of Medicine, University of Toronto, Faculty of Health, York University (CPP), Toronto, Ontario; Spasticity Research Program, West Park Healthcare Centre, and Division of Physiatry, Faculty of Medicine, University of Toronto (FI), Toronto, Ontario; Spasticity Research Program, West Park Healthcare Centre, and Division of Physiatry, Faculty of Medicine, University of Toronto, Toronto, Ontario (CB)

Received SePtember 29, 2014. Final Revisions Submitted April 8, 2015. Correspondence to: Chris Boulias, Spasticity Research Program, 82 Buttonwood Ave., Toronto, ON, Canada M6M 2J5. Email: chris.boulias@westpark.org. 
complete the survey. Although the questionnaire has not been validated or tested for reliability, it has been previously used to assess spasticity-related literacy. ${ }^{3}$

\section{RESULTS}

\section{Response Rate}

Of the 2795 emails sent to physicians, 564 bounced or were incorrect. Of the 2231 emails successfully sent, 111 physicians completed the online questionnaire (5.0\% response rate). Response rate was similar across all provinces; 84 of the respondents were Canada-trained, whereas 27 were internationally trained (18 from developing economies; 67\%). The average $( \pm$ standard deviation) number of years of experience practicing in our group was $28 \pm 11$. Male to female ratio was $65 / 46$ and the ratio of those trained in Canada to those trained outside Canada was $82 / 29$.

\section{Spasticity Health Literacy Across Canada}

Only $25 \%$ of all respondents were able to correctly identify the definition of spasticity, $32 \%$ of physicians felt adequately trained to recognize spasticity in the community, and $13 \%$ of all respondents reported having adequate knowledge regarding beneficial treatments for spasticity. Spasticity can cause functional problems, but also be functionally important. Although $87 \%$ physicians felt their knowledge of spasticity treatments was deficient, assessment of spasticity and the functional impact needs to be tested by physicians with expertise in spasticity management. No significant differences were found between internationally trained and Canada-trained physicians with regard to providing the correct definition of spasticity, reporting feeling adequately trained to recognize spasticity, or having adequate knowledge of the beneficial treatments.

Chi-squared analyses were performed and results indicated that Canada-trained physicians were 2.6 times more likely than internationally trained physicians to correctly identify the treatment for focal spasticity. Furthermore, Canada-trained physicians were 2.3 times more likely than internationally trained physicians to identify the correct treatment for generalized spasticity $(p<0.05)$. Gender and years of experience did not influence any of the answers in the study.

Figure 1 illustrates a breakdown of the responses when physicians were asked to choose the definition of spasticity; 1A-C contain responses by physicians when asked to choose the best possible treatment option for either generalized, focal, or unresponsive generalized (at low doses of oral medications) types of spasticity, respectively.

\section{Spasticity Resource Availability}

Among all of the physicians surveyed, $66 \%$ reported problems with the availability of spasticity resources in their communities or larger centers nearby. When the physicians were asked to list the spasticity resources available in their respective communities, $14.4 \%$ reported patient support groups, $84 \%$ reported physiotherapy, $68 \%$ reported occupational therapy, $73 \%$ reported experts in spasticity management, and 34\% reported the presence of surgeons. The reported availability of spasticity resources was not significantly different when the respondents were stratified according to location of training, years of experience, or gender; see Table 1 for a summary of responses.

\section{Discussion}

\section{Spasticity Health Literacy}

The limited spasticity literacy, attributable to a lack of adequate training, was found in a small cohort of family physicians with equivalent representation from all provinces of Canada. Although the representation was similar across provinces, this sample does not represent all family physicians in Canada. The majority of physicians surveyed were unable to identify the

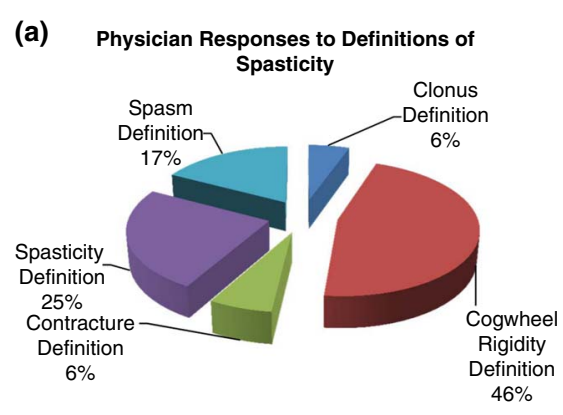

(c)

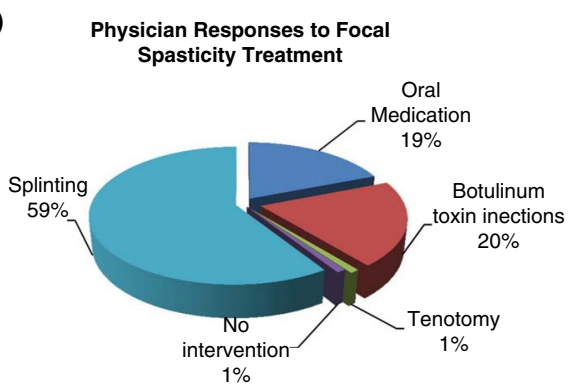

Figure 1: Summary of physician responses.

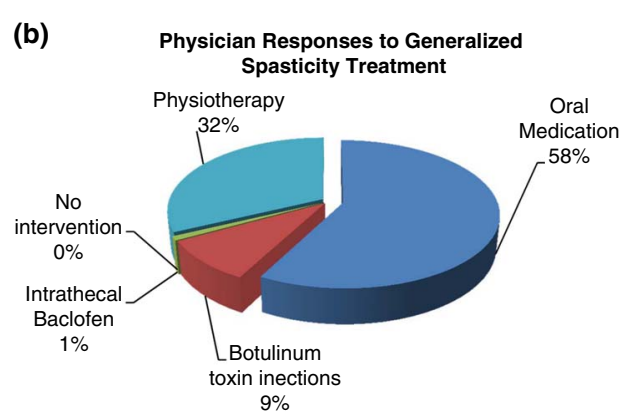

(d) Physician Responses to Unresponsive Generalized

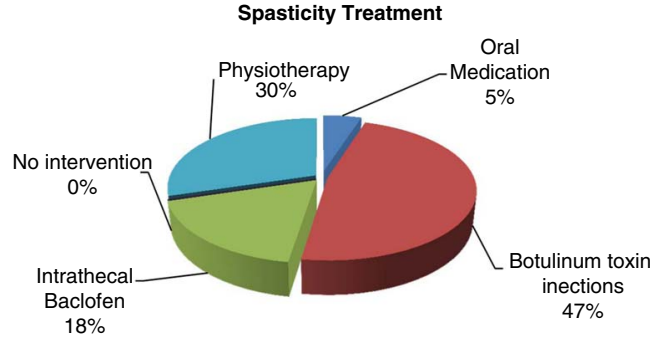




\section{Table 1: Summary of responses}

\begin{tabular}{|c|c|}
\hline Question & Response (\%) \\
\hline \multicolumn{2}{|l|}{ Chosen definition of spasticity } \\
\hline Clonus & 6 \\
\hline Cogwheel rigidity & 46 \\
\hline Contracture & 6 \\
\hline Spasticity & 25 \\
\hline Spasm & 17 \\
\hline \multicolumn{2}{|l|}{ Treatment of focal spasticity } \\
\hline Oral medication & 19 \\
\hline Botulinum toxin $\mathrm{A}$ injections & 20 \\
\hline Tenotomy & 1 \\
\hline No intervention & 1 \\
\hline Splinting & 59 \\
\hline \multicolumn{2}{|c|}{ Adequately training to recognize spasticity } \\
\hline Yes & 32 \\
\hline No & 68 \\
\hline \multicolumn{2}{|c|}{ Problem with the availability of spasticity treatments } \\
\hline Yes & 66 \\
\hline No & 34 \\
\hline \multicolumn{2}{|l|}{ Treatment of generalized spasticity } \\
\hline Oral medication & 58 \\
\hline Botulinum toxin A injections & 9 \\
\hline Intrathecal baclofen & 1 \\
\hline No intervention & 0 \\
\hline Physiotherapy & 32 \\
\hline \multicolumn{2}{|c|}{ Treatment of unresponsive generalized spasticity } \\
\hline Oral medication & 5 \\
\hline Botulinum toxin A injections & 47 \\
\hline Intrathecal baclofen & 18 \\
\hline No intervention & 0 \\
\hline Physiotherapy & 30 \\
\hline \multicolumn{2}{|c|}{ Adequate knowledge about spasticity treatments } \\
\hline Yes & 13 \\
\hline No & 87 \\
\hline
\end{tabular}

correct definition of spasticity (Figure 1A). Although the majority of responders correctly identified effective pharmacological strategies (Figure 1B) to manage generalized spasticity using oral medications (58\%), only about one-third identified physiotherapy as an approach to manage spasticity. There is some evidence to indicate the benefit of spasticity management using physiotherapy exercises, but only in upper and not lower limbs. ${ }^{4}$ The majority of physicians (80\%; Figure 1C) were unable to identify botulinum toxin injections as the evidenced-based effective approach for focal spasticity management and inaccurately identified splinting as a method to manage focal spasticity. Recent evidence indicates that long-term use of splints is required to minimize spasticity, but long-term use is uncomfortable and thus not feasible. ${ }^{5}$ Unresponsive generalized spasticity is best treated with intrathecal baclofen injections especially in younger adults with spinal cord injury. ${ }^{2}$ However, only $18 \%$ of responders correctly identified treatment for unresponsive generalized spasticity management (Figure 1D).

Significant knowledge discrepancies between Canadian and internationally trained physicians may be related to differences in curriculum content. However, given the small sample of internationally trained physicians $(n=27)$, we acknowledge that the results obtained in the study may not be generalizable to the internationally trained population as a whole. The deficiencies in knowledge ranged from identification of spasticity to effective management. These deficits may impact the general well-being and functionality of the patient and may create financial and social burden arising from increased caregiving requirements.

\section{Resource Availability Across Canada}

In addition to problems patients may face in having their spasticity recognized in the primary care setting, once the diagnosis has been made there is the further obstacle of scarce resources to overcome. This was demonstrated by the finding in our study that $66 \%$ of family physicians reported problems with the availability of spasticity treatments in their respective communities. This included patient access to physiotherapy, occupational therapy, experts in spasticity management, surgeons, and patient support groups. A lack of clinical knowledge combined with lack of access to adequate resources may severely impact patient outcomes in the long run.

\section{Study Limitations and Future Directions}

The overall response rate for this study was low, with a rate of $5 \%$. The email addresses of family physicians were obtained from Scott's Medical Directory, and it was not possible to verify whether the email addresses were correct or updated. It is possible that many of the email addresses were inaccurate or were no longer in use. It is also possible that the survey invitations ended up in junk folders and were therefore never delivered to the intended recipient's inbox. Because we were unable to differentiate between those who received the survey and those who did not, we were unable to determine whether nonresponders differed from responders. With the very low response rate and the inability to compare differences between responders and nonresponders, generalizability of the findings in this study is limited.

Although a prior study was limited to the province of Ontario, this study extended the scope to encompass all of Canada. However, whereas the previous study stratified its results to compare urban versus rural access to spasticity resources, this study did not compare those two variables. Rather, the current study stratified physicians according to location of training, years of experience, and gender. Furthermore, the internationally trained physicians were not segregated according to country of training, but instead grouped together in a single internationally trained category. The sample size placed limitations on our ability to draw conclusions about international training programs on an individual basis. A higher response rate would allow for further stratification of results according to country of training and province of practice. 


\section{Conclusion}

The results of this study show that spasticity literacy is low among all surveyed Canadian family physicians and significantly lower among those trained outside Canada. Because years of practice experience did not significantly improve physician knowledge, further education and training is required during medical school and family practice residency and for those in practice via continuing medical education programs.

\section{Disclosures}

The views expressed in the article are solely the opinions of the authors. None of the authors declare any conflicts of interest in the undertaking of this study. JG has nothing to disclose. $\mathrm{CP}$ has received past grant support from Allergan, Inc. FI has is a consultant and has received honoraria and speaker's fees from Allergan and Merz Pharma. CB is a consultant for and has received honoraria from Allergan, Inc., and Merz Pharma.

\section{SUPPLEMENTARY MATERIAL}

To view supplementary material for this article, please visit http://dx.doi.org/10.1017/cjn.2015.271

\section{REFERENCES}

1. Lance J. Symposium synopsis. In Feldman R, Young R, Koella W editors. Spasticity disordered motor control. Chicago Year Book Medical Publishers; 1980, p. 485-94.

2. Bhakta BB. Management of spasticity in stroke. Br Med Bull. 2000;56:476-85.

3. Canning M, Phadke C, Ismail F, Boulias C. A survey of rural and urban family physicians: analysis of spasticity health literacy and availability of appropriate spasticity care. Univ Toronto Med J. 2013;90:114-8.

4. Wolf SL, Milton SB, Reiss A, Easley KA, Shenvi NV, Clark PC. Further assessment to determine the additive effect of botulinum toxin type A on an upper extremity exercise program to enhance function among individuals with chronic stroke but extensor capability. Arch Phys Med Rehabil. 2012;93:578-87.

5. Andringa A, van de Port I, Meijer JW. Long-term use of a static hand-wrist orthosis in chronic stroke patients: a pilot study. Stroke Res Treat. 2013;2013:546093. 